\title{
ESTUDIANTES CON DISCAPACIDAD Y LA ORIENTACIÓN VOCACIONAL EN SECUNDARIA: UNA REVISIÓN SISTEMÁTICA CON VOSVIEWER
}

\author{
Greisy González Cedeño \\ Universidad Especializada de las Américas. Panamá \\ greisy3028@gmail.com \\ Brizida Hernández Sánchez \\ Universidad Especializada de las Américas. Panamá \\ hernandez.brizeida@gmail.com
}

Recepción Artículo: 10 febrero 2020

Admisión Evaluación: 4 marzo 2020

Informe Evaluador 1: 13 marzo

Informe Evaluador 2: 17 marzo 2020

Aprobación Publicación: 20 abril 2020

\section{RESUMEN}

El proceso de orientación debe facilitar que la persona desarrolle estrategia para comprender la ambigüedad y la aceleración de los cambios del entorno laboral. El propósito de este artículo es analizar la información existente y determinar a partir de las revisiones sistemáticas de la literatura que se han realizado sobre los programas de orientación vocacional y la relación con estudiantes con discapacidad, a que conclusiones se ha llegado, las lagunas que persisten y cuáles son los siguientes pasos para seguir a fin de encontrar mejoras en dichos programas, así como identificar las barreras a las que estudiantes e instituciones pueden enfrentarse.

Por tanto, Ios principales objetivos están enmarcados en identificar las diferentes perspectivas teóricas que han sido estudiadas sobre los programas de orientación vocacional educativa y destacar las conexiones entre ellos; buscar tendencias emergentes y determinar las lagunas al comparar las diferentes investigaciones, y finalmente, determinar de las conclusiones a las que se ha llegado, si posteriormente ha habido algún cambio importante que permitiera el avance científico en esta área.

Además, se van a analizar algunos datos bibliométricos, para situar la investigación dentro de la educación inclusiva secundaria.

Se recopilaron los datos bibliográficos y se analizamos a través de técnicas de co-cita con los siguientes objetivos específicos:

- Identificar las diferentes perspectivas teóricas que han sido estudiadas sobre la los programas de orientación vocacional y destacar las conexiones entre ellos;

- Buscar tendencias emergentes y determinar las lagunas al comparar los diferentes investigaciones, y

- Determinar posibles factores críticos que permitan el avance científico en el área 


\title{
ESTUDIANTES CON DISCAPACIDAD Y LA ORIENTACIÓN VOCACIONAL EN SECUNDARIA: UNA REVISIOON SISTEMÁTICA CON VOSVIEWER
}

En este sentido, este artículo integra las revisiones sistemáticas que se han realizado alrededor del tema de la orientación vocacional.

Palabras clave: curricula; estudiantes; instrucción; barreras; estudiantes con discapacidad; human capital

\begin{abstract}
Students with disabilities and vocational guidance in secondary schools: a systematic review with vosviewer. The orientation process should facilitate the person to develop a strategy to understand the ambiguity and acceleration of changes in the work environment. The purpose of this article is to analyze the existing information and determine from the systematic reviews of the literature that have been carried out on vocational guidance programs and the relationship with students with disabilities, to which conclusions have been reached, the gaps that persist and what are the next steps to follow in order to find improvements in these programs, as well as identify the barriers that students and institutions may face.

Therefore, the main objectives are framed in identifying the different theoretical perspectives that have been studied on educational vocational guidance programs and highlighting the connections between them; look for emerging trends and determine gaps when comparing the different investigations, and finally, determine from the conclusions that have been reached, if there has subsequently been any important change that allowed scientific progress in this area.

In addition, some bibliometric data will be analyzed to situate the research within inclusive secondary education.

Bibliographic data was collected and analyzed through co-appointment techniques with the following specific objectives:

Identify the different theoretical perspectives that have been studied about vocational guidance programs and highlight the connections between them;

Look for emerging trends and determine gaps by comparing different investigations, and

Determine possible critical factors that allow scientific progress in the area

In this sense, this article integrates the systematic reviews that have been carried out around the topic of vocational guidance.
\end{abstract}

Keywords: curriculum; students; instruction; barriers; students with disabilities; human capital

\section{INTRODUCCIÓN}

En la educación secundaria algunos estudiantes toman decisiones equivocadas en su carrera debido a la ignorancia, la inexperiencia, la presión de los compañeros, el consejo de amigos, padres y maestros, o etiquetas adjuntas con ciertos tipos de trabajo sin el conocimiento adecuado (Chirenjeevi Reddy \& Rajaran, 2014).

La preocupación creciente por la necesidad de encontrar sitio en el ámbito laboral para la gran cantidad de estudiantes que va en aumento, significa un reto mayúsculo para las instituciones educativas.

La escuela ofrece oportunidades para establecer relaciones sociales fuera de la familia, principalmente con sus compañeros, pero también con adultos que pueden actuar como modelos a seguir y brindar orientación y apoyo en relación con el futuro. Por lo cual, los estudiantes deben tomar decisiones de vida, las cuales pueden ser una limitante importante. Se puede esperar que las posibilidades de parte de la escuela para actuar como un escenario social favorable para los estudiantes estén vinculadas a sus capacidades de proporcionar "efectividad escolar" (Alma, Brolin Låftmanb, Sandahlc, Modinb, 2019).

Además, tiene que analizarse desde un contexto que involucra una gran cantidad de estímulos que provienen del efecto de la globalización (Mouzakitis, 2010), tomando en cuenta minorías estudiantiles como personas adultas 0 con discapacidad (Canhaa, Simõesa, Owens \& Matosa, 2012) 0 enfermedades mentales (Thanapalan, Suleiman \& Mohd Natar, 2016). 


\section{CRECIMIENTO PSICOLÓGICO Y AFRONTAMIENTO DE LA MADUREZ}

Para UNESCO (2014) la educación secundaria ha crecido en demanda y el interés por el desarrollo de las capacidades profesionales, sobre todo en un contexto de desempleo creciente de los jóvenes y el proceso de cualificación.

El sistema educativo da acceso a los niños y jóvenes con necesidades educativas especiales, ofreciendo educación bilingüe intercultural para grupos originarios; programas educativos para niños y jóvenes en comunidades de riesgo y programas de alfabetización para jóvenes y adultos. Una educación inclusiva en secundaria debe reunir lo mejor de la enseñanza para cada estudiante y su familia. Llevar una teoría ecológica funcional al aula propone bases sólidas para todo profesor. Para lograrlo, se requiere una educación individualizada (Rico 2010; Perkins International, 2016). Este enfoque beneficia a los estudiantes con discapacidad en la escuela que presentan retos múltiples o disparidades que no son invisible.

\section{MÉTODO}

Los autores decidieron diseñar un artículo científico usando la revisión sistemática, la cual sintetiza resultados de investigaciones primarias (Beltrán \& Oscar, 2005) que ayudan a determinar el estado del arte de un campo de estudio (Sánchez-Meca, 2010). Se utilizaron filtros de control basados en evidencias científicas como la sistematización para la selección y el rechazo de artículos. Además, se tomaron decisiones previas de criterios explícitos, aplicados a artículos para delimitar el tema y cumplir los objetivos. Sólo se utilizaron artículos indexados y open Access.

\section{PROCEDIMIENTO}

Este artículo de revisión sistemática siguió los siguientes pasos: 1. Selección de los artículos 2. Extracción del método para extraer información relevante y 3. Diseño de una tabla con la síntesis de los hallazgos más relevantes encontrados. En la búsqueda de artículos y revisiones realizadas, se utilizó el Social Science Citation Index (SSCI), la base de Science Direct. Se escogió por la parte científica de SSCI que aglutina las principales revistas, documentos y artículos académicos en open Access. Se utilizaron estas dos bases de datos para realizar un proceso de selección que determinara de mejor forma los conceptos que querían revisarse.

Sobre la estrategia de búsqueda en la base de datos se utilizó el procedimiento sistemático que se detalla a continuación:

Se seleccionaron los términos de búsquedas "education", "vocational programs", "orientation". "students with disabilities"

En una primera búsqueda se identificaron 129 documentos de Science Direct. La información se extrajo del año 2010 a 2020 (Ver Anexo 1). Paso seguido se procedió a analizar, eliminar y seleccionar la información. El siguiente paso fue revisar cada artículo para comprobar su relación con la temática por lo que nos resultó 12 documentos. De esta manera nos aseguramos tener respuesta a las preguntas de investigación.

Se realizó una base de datos en Excel (Ver anexo 2) donde se incluyeron los artículos resultantes de la última revisión de la literatura. 
ESTUDIANTES CON DISCAPACIDAD Y LA ORIENTACIÓN VOCACIONAL EN SECUNDARIA:

UNA REVISIÓN SISTEMÁTICA CON VOSVIEWER

Figura 1: Evolución histórica del estudio de programas de orientación vocacional

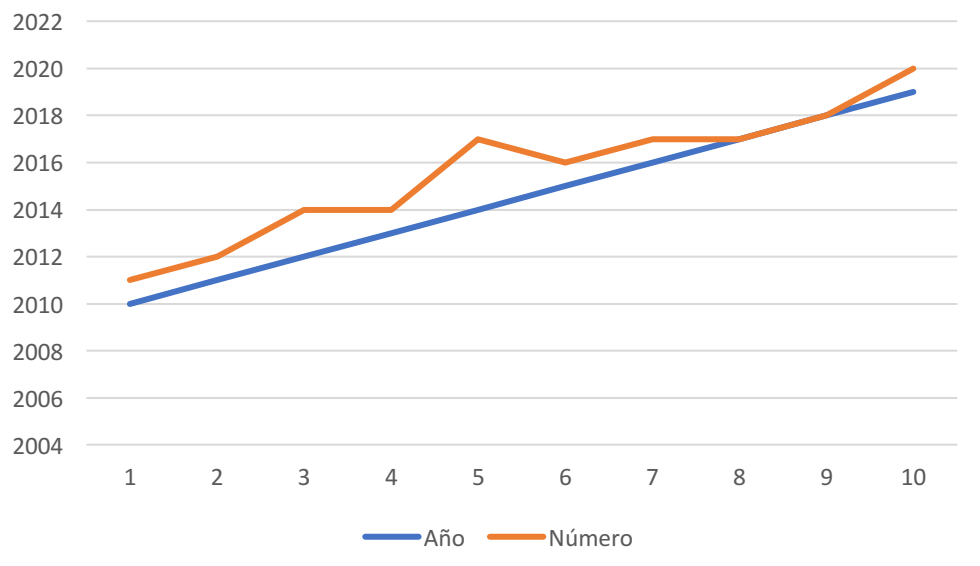

Fuente: Elaboración propia (2020).

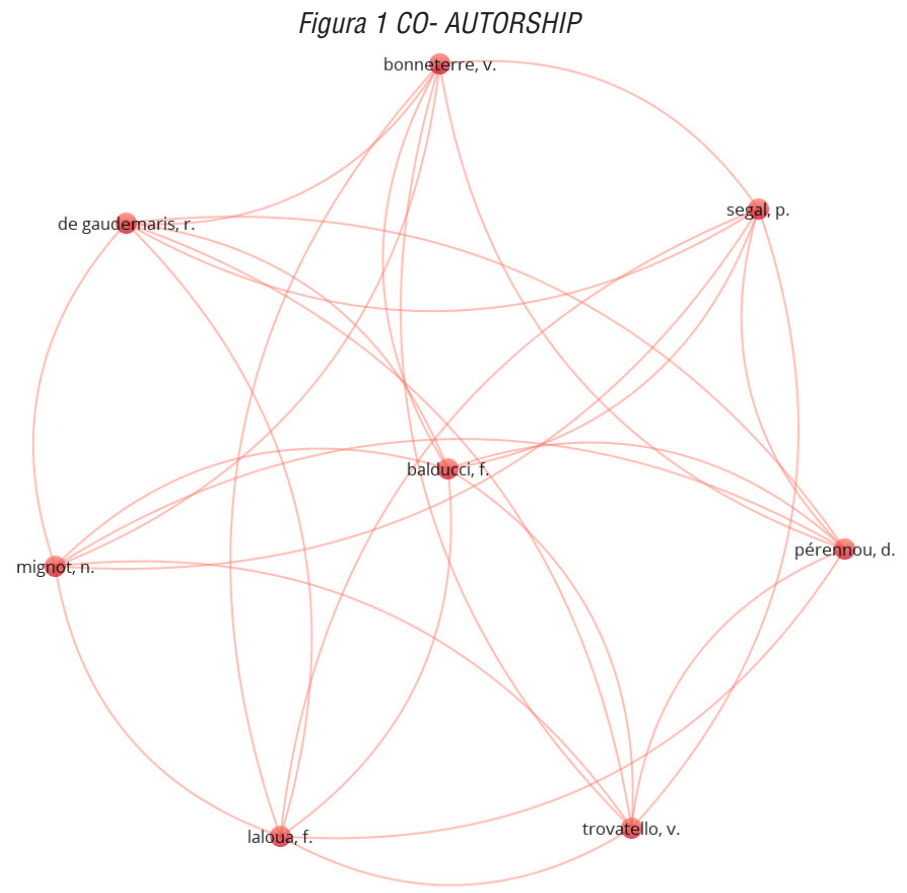

Fuente: VosViewer 
Figura 2 Co ocurrence

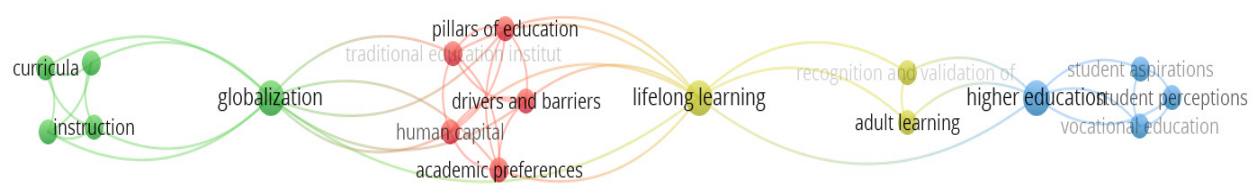

Fuente: VosViewer

\section{RESULTADOS}

A partir de la revisión sistemática, se encontraron 4 clústers que se explican a continuación:

Cluster 1: "academic preferences", "drivers and barriers", "human capital", "pillars of education", "traditional education institution" Uno de los propósitos del sistema educativo formal es mediar los valores esenciales. La democracia es uno de esos valores. Los documentos de política educativa de nivel global nivel nacional y local también dan prioridad al aprendizaje de cómo lograr el desarrollo sostenible (Torbjörnsson, 2011).

La Educación Básica de calidad sienta las bases necesarias para el aprendizaje. Hay un periodo de la vida que es fundamental para la cualificación y gestión de las competencias, es el periodo de 12 a los 18 años, es decir la educación secundaria. Esta articulación en el sistema educativo con las políticas entre educación y el trabajo tienen un importante rol que cumplir en la vida del joven. Por ello, es necesario que las ofertas educativas y el funcionamiento de los programas de formación, permitan a los jóvenes con discapacidad enfrentar en mejores condiciones de participación y aprendizaje desarrollando al máximo sus competencias y destrezas en el momento de su inserción laboral.

Las escuelas pueden llevar a cabo una orientación previa a la práctica para sus estudiantes, de modo que las expectativas y los procedimientos puedan ser presentados y discutidos (Aquino, 2014).

En la educación secundaria se produce un sesgo de acceso a las oportunidades entre los egresados al mercado laboral y los que están por egresar. Frente a este panorama se suma la escasa enseñanza de habilidades socioemocionales y de datos que sirvan para orientar la oferta y la demanda de formación profesional. Para muchos estudiantes, la educación secundaria es el nivel de escolaridad más elevado que va a alcanzar antes de incorporarse al mercado laboral (Castro; Carnoy).

Los programas educativos implementados a través de una combinación de mecanismos organizativos: extensión, divisiones, unidades de educación continua y para jóvenes adultos, escuelas de estudio profesional, programas de educación ejecutiva y unidades de educación a distancia (Chitiva, 2012).

Entre varias curriculas actuales, se hace énfasis en la búsqueda de valores, los cuales son necesarios para ayudar al estudiante a dirigir su vocación de una manera ética. Otra fuente de valores comunes a nivel público es la ideología. Una función de una ideología es imprimir narraciones sobre el futuro (Torbjörnsson, 2011). El concepto de ethos escolar se refiere a las normas, valores, actitudes y comportamientos que caracterizan los patrones de interacción de docentes y estudiantes en una escuela determinada (Alma, Brolin Låftmanb, Sandahlc, Modinb, 2019).

Es necesario tomar en cuenta el entorno educativo es un entorno en el que el desarrollo positivo de los estudiantes puede reflejarse fácilmente. Sin embargo, no todos los sistemas educativos son iguales ni intentan satisfacer las necesidades de los adolescentes de la misma manera (Orejudo-Hernández, Aparicio-Moreno \& CanoEscoriaza, 2013).

Cluster 2: "curricula", "employees qualifications", "globalization", "instruction", market research". Las universidades y otras instituciones de educación superior son fundamentales para el desarrollo y la innovación del capital humano y pueden hacer mucho para ayudar a la fuerza laboral a ser más innovadora y competitiva a nivel mundial. Sin embargo, para hacer esto necesitan cambiar, y en algunos casos cambian dramáticamente. Las universidades y otras instituciones de educación superior deben salir de su zona de confort y acompañar al estudiantes y dar accesos a estudiantes con discapacidad (Chitiva, 2012). 


\section{ESTUDIANTES CON DISCAPACIDAD Y LA ORIENTACIÓN VOCACIONAL EN SECUNDARIA: UNA REVISIÓN SISTEMÁTICA CON VOSVIEWER}

La globalización se ha convertido en uno de los temas cruciales de discusión y preocupación en los últimos tiempos, ya que tiene un impacto decisivo en nuestras vidas. Por lo tanto, si queremos competir en el escenario global y enfrentar los desafíos de la globalización con éxito, necesitamos realizar mejoras significativas en términos de maximizar la efectividad de la educación vocacional y la instrucción de capacitación basada en el diseño específico de los planes de estudio (Mouzakitis, 2010).

La diversidad de estos programas y sus operaciones únicas propagaron organizaciones completamente nuevas dentro de las universidades tradicionales. La idea de formar socios de capacitación puede asignar mentores prácticos que se encargarán de la capacitación práctica de los estudiantes y dar retroalimentación a los estudiantes periódicamente. También pueden llevar a cabo una sesión informativa o una orientación antes del inicio de la capacitación práctica para que los estudiantes puedan recibir información sobre la organización, los protocolos y otras preocupaciones operativas únicas (Aquino, 2014).

Sin embargo, la llegada de la globalización ha exigido mercados laborales más especializados, niveles más altos de habilidades y una educación vocacional diversificada que no contempla los estudiantes con discapacidad. Durante años, ha habido una creciente conciencia de que la educación general es a menudo demasiado académica y no prepara a los jóvenes adecuadamente para el mundo laboral y en el caso del estudiante con discapacidad sigue están en desventaja. En comparación con la educación general, la educación y formación profesional tiene un vínculo más cercano y directo con el desarrollo económico y profesional. Como consecuencia de este crecimiento, las organizaciones y las empresas deben responder de manera estratégica a los cambios que tienen lugar para beneficiarse del crecimiento económico (Mouzakitis, 2010).

Los directivos pueden discutir con posibles socios de capacitación, hacer alianzas estratégicas y diseñar programa de orientación vocacional que sea aceptable para ambos e incluyentes, de modo que haya una experiencia de capacitación práctica significativa para los estudiantes. La asignación de un representante de la escuela para monitorear la capacitación práctica de los estudiantes garantizaría que se logren los objetivos de la práctica (Aquino, 2014).

La orientación futura se puede dividir en cognitiva, motivacional y un componente afectivo, donde el uno afectiva, que se refiere principalmente a los sentimientos de una persona en términos de optimismo / pesimismo respecto al futuro, es tal vez el más intuitivo y el aspecto más comúnmente estudiada (Alma, Brolin Låftmanb, Sandahlc, Modinb, 2019).

Cluster 3: "higher education", "students aspirations", "students perceptions", "vocational education"

El cluster hace referencia a que en cualquier proceso de educación superior, para el continuo desarrollo integral del estudiante, la elección correcta del curso y el aprendizaje efectivo durante el programa son muy importantes y dependen principalmente del interés del estudiante, el conocimiento del dominio, las percepciones y las aspiraciones. La elección de carrera se ha convertido en una tarea compleja con el advenimiento de la tecnología de la información, el surgimiento de una revolución post industrial con diferentes campos y variedad de trabajos (Chirenjeevi Reddy \& Rajaran, 2014).

Dentro de este campo de la Formación Profesional también hay dos perfiles distintivos de estudiantes: aqueIlos que han completado con éxito la Educación Secundaria requerida y desean completar la formación profesional y aquellos que han sido admitidos en la Formación Profesional a través de los Programas de Calificación Profesional Inicial (PCPI) sin haber completado con éxito la educación secundaria (Orejudo-Hernández, AparicioMoreno \& Cano-Escoriaza, 2013).

La elección de carrera está influenciada por múltiples factores que incluyen la personalidad, los intereses, el autoconcepto, la identidad cultural, la globalización, la socialización, el modelo a seguir, la preparación de los padres, el apoyo social y los recursos disponibles, como información y fortalezas financieras. Una vez que los estudiantes se unen al curso elegido, el colegio es el principal responsable de comprender los factores mencionados y acomodar el aprendizaje requerido para que los estudiantes cumplan con sus aspiraciones (Chirenjeevi Reddy \& Rajaran, 2014) 
La mayoría de los estudiantes sin discapacidad siguen estos cursos, ya que las principales habilidades requeridas para tener éxito en estas actividades profesionales son la creatividad, el dibujo, la presentación, la espontaneidad y la buena comunicación con horas de trabajo prolongadas. Excepto por las habilidades de comunicación, los estudiantes en riesgos vulnerables poseen las habilidades restantes en gran medida. Por lo tanto, al alentar y apoyar a los estudiantes en esta situación, los buenos profesionales de la moda pueden evolucionar debido a sus habilidades innatas que se adaptan a los programas de moda adecuado (Chirenjeevi Reddy \& Rajaran, 2014).

Cluster 4: "adult learning", "lifelong learning", "recognition and validation": El cluster busca explicar inicialmente lo que se considera "adulto", Io cual, puede variar dependiendo de cada cultura y la normativa vigente en cada país o región (Németh, 2014). De acuerdo a ello, deberán establecerse programas educativos diseñados expresamente para este tipo de población que tiene sus propias necesidades y demandas de tiempo, espacio y motivaciones.

El alcance, las perspectivas y las oportunidades de empleo son enormes, ya que la industria se ha extendido por muchos campos (Petrovic-Randalovic \& Miletic, 2012), promoviendo capacidades en las personas como la creatividad, innovación y búsqueda de programas vocacionales interdisciplinarios y materias afines deben desarrollar una mentalidad abierta y una personalidad equilibrada para un aprendizaje efectivo para enfrentar los desafíos en el entorno dinámico de la vida real. Para el desarrollo continuo y general del estudiante en educación, su estado actual, conocimiento del dominio, percepciones y aspiraciones juegan papeles importantes (PetrovicRandalovic \& Miletic, 2012).

Entre los programas vocacionales más estudiados está la educación de moda es una de las corrientes de educación vocacional de rápido crecimiento y prosperidad en todo el mundo. Tiene rápidamente expandido durante las últimas dos décadas. Por otro lado, en la educación relacionada con la construcción, el desarrollo del liderazgo sigue siendo una característica escasa en los planes de estudio de la educación superior. Sin embargo, la necesidad crítica de un "buen" liderazgo, en todos los niveles de las organizaciones relacionadas con la construcción, se destaca cada vez más en la investigación y la práctica de la gestión de la construcción (Räisänen, Josephsson \& Luvö, 2015).

\section{DISCUSIÓN Y CONCLUSIONES}

La educación secundaria ha crecido en demanda y el interés por el desarrollo de las capacidades profesionales, sobre todo en un contexto de desempleo creciente de los jóvenes y el proceso de cualificación. El sistema educativo da acceso a jóvenes con necesidades educativas especiales, ofreciendo educación que aún no responde a las demandas competitivas.

Sin duda la actitud vocacional depende del núcleo académico de las instituciones educativas que buscan el mejor futuro de sus estudiantes, sin exclusividad estudiantes con o sin discapacidad de algún rango de edad específico. En la educación superior convencional se encontraron diferentes parámetros que influyen en la elección de carrera y la entrega del plan de estudios, su influencia y cómo los estudiantes reconocen estos cursos desde el punto de vista profesional, cuáles son sus antecedentes, sus intenciones y aspiraciones adecuado (Chirenjeevi Reddy \& Rajaran, 2014).

Las limitantes del estudio recaen en la búsqueda de términos que agrupen un núcleo de estudios en específico. Se recomienda hacer un análisis de programas vocacionales dependiendo del área de estudios que se busque analizar. También, tomar en cuenta a todo tipo de estudiantes (adultos, personas con discapacidades) (Canhaa, et. al., 2012).

Por otro lado, deben tomarse en cuenta programas que evalúen la orientación vocacional brindando correcta retroalimentación a la institución para completar el ciclo y además, tomar en cuenta posibles nichos de oportunidad para el fortalecimiento de la perspectiva que maneje la institución. Por tanto, la retroalimentación de profesores a alumnos dentro de programas de orientación vocacional es fundamental (Carvalhoa, Conboya, \& Martinsa, 2014). 


\section{ESTUDIANTES CON DISCAPACIDAD Y LA ORIENTACIÓN VOCACIONAL EN SECUNDARIA: UNA REVISIOON SISTEMÁTICA CON VOSVIEWER}

En este sentido, los cambios que se han producido en el mercado laboral afectan de una forma bastante sobresaliente. La elección profesional es el resultado de un proceso, por lo que es necesario una práctica educativa continuada, que facilite el alumnado vaya construyendo su propio proyecto profesional personal. La orientación vocacional como proceso educativo persigue la adquisición por parte de la persona habilidades, destrezas y actitudes para orientarse (auto orientación). El proceso de orientación debe facilitar que la persona desarrolle estrategia para comprender la ambigüedad y la aceleración de los cambios del entorno laboral. Y tomar conciencia de los recursos de los que dispone para enfrentarse a esta situación. La prevalencia del desarrollo de habilidades y actitudes sobre la adquisición de informaciones puntuales posibilita la independencia de la persona.

El modelo ofrece una propuesta curricular que busca lograr en cada persona la no discriminación que va desde una representación personal y profesional con una toma de conciencia de la desigualdad y de los factores que llevan a tener una imagen de sí mismo. Conseguir en cada alumno elabore un proyecto profesional personal en el que se consideren, junto a otras limitaciones, las que vienen promovidos de alumnos serán diferentes por ser personas distintas, no por ser desiguales.

Sobre los contenidos en que sea capaz de indagar y buscar activamente, en el que se dispongan en ejercicio los procesos y operaciones cognitivas implicadas en la secuencia del desarrollo vocacional. Este proceso debe favorecer la autonomía a través de la participación activa del proceso de orientación, va a determinar por sí mismo.

\section{Recomendaciones}

- El documento identificó varios programas de educación vocacional así como conceptos relacionados y la relación con los estudiantes con discapacidad.

Limitaciones / implicaciones de la investigación

- Inevitablemente, los hallazgos del análisis tienen limitaciones relacionadas con el diseño de la investigación, las bases de datos utilizadas y los métodos bibliométricos aplicados dentro de la revisión sistemática de estos constructos.

Implicaciones prácticas

- Desde una perspectiva práctica, la investigación impacta en la construcción de teorías, el desarrollo histórico del constructo y las fortalezas y debilidades dentro de la investigación que aún persisten.

Originalidad / valor

- Este estudio describe el notable desarrollo de la literatura sobre co-creación y muestra la estructura latente subyacente a sus diferentes corrientes de investigación. Para el mejor conocimiento, este estudio es el primero en determinar las frecuencias de citación de las bases de datos de Science direct.

Nuestro agradecimiento a la Secretaria Nacional de Discapacidad (SENADIS,2017) Panamá, que ha sido un pilar fundamental en los resultados de investigación desde la Universidad Especializada de las Américas en la Maestría de Políticas Públicas.

\section{REFERENCIAS BIBLIOGRÁFICAS}

Alm, S., Brolin Låftmanb, S., Sandahlc, J., \& Modinb B. (2019) School effectiveness and students' future orientation: A multilevel analysis of upper secondary schools in Stockholm, Sweden. Journal of Adolescence 70, 62-73. doi: 10.1016/j.adolescence.2018.11.007

Aquino, M. C. G. (2014). More hours, more areas, better practicum training....? Procedia - Social and Behavioral Sciences, 144. 264-272. doi: 10.1016/j.sbspro.2014.07.295.

Canhaa L., Simõesa,C., Owens, L., Matosa, M. (2012). The importance of perceived quality-of-life and personal resources in transition from school to adult life. Procedia - Social and Behavioral Sciences 69. 1881-1890. doi: 10.1016/j.sbspro.2012.12.141.

Carvalhoa, J. S., Conboya, J. \& Martinsa, D. (2014). Exploring Differences in Students Perceptions. Procedia Social and Behavioral Sciences 159. 168-173. doi: 10.1016/j.sbspro.2014.12.351 
Castro, C. D. M., Carnoy, M., y Wolff, L. (2000). Las escuelas de secundaria en América Latina y el Caribe y la transición al mundo del trabajo. Inter-American Development Bank.

Chirenjeevi Reddy G. \& Rajaran N. J. (2014). Career aspirations and background of students opting for fashion education courses in India., 176, 952-960. doi: 10.1016/j.sbspro.2015.01.564

Chitiva, C. A. (2012). Lifelong learning challenges and opportunities for traditional universities. Procedia- Social and Behavioral sciences, 46, 1943-1947.

Mouzakitis, G. S. (2010). The role of vocational education and training curricula in economic development. Procedia Social and Behavioral Sciences 2. 3914-3920. doi:10.1016/j.sbspro.2010.03.616.

Németh, B. (2014) Research and development of adult education through higher education institutions: a challenge and perspective for better adult learning and education. Procedia - Social and Behavioral Sciences 142, $97-103$.

Orejudo-Hernández, S., Aparicio-Moreno, L., \& Cano-Escoriaza, J. (2013). Personal competencies of spanish students pursuing different academic careers. contributions and reflections from positive psychology. Journal of Behavior, Health \& Social Issues, 5(2), 63-78. doi:10.5460/jbhsi.v5.2.42253

Räisänen, C., Josephsson P. E., \& Luvö, B. (2015) Stakeholders' views and experiences of leadership education in construction: suggestions for improvements. Procedia Economics and Finance 21. 540-547.

Thanapalan, C. K. K. , Suleiman, M., Mohd Natar, A. K. (2016). Work Environmental Support from Small Industry's Employer Perspectives for Workers with Mental IIIness. Procedia - Social and Behavioral Sciences 222. 423-431.

Torbjörnsson, T. (2011). Attitudes to sustainable development among Swedish pupils. Pr Christine Räisänen*, Per-Erik Josephsson and Bert Luvöocedia- Social and behavioral sciences, 15, 316-310. doi:10.1016/j.sbspro.2011.03.093.

Rico, A. P. (2010). Políticas de educación inclusiva en América Latina Propuestas, realidades y retos de futuro. Revista de educación inclusiva, 3(2). 
